\title{
Embedding DRT in a Situation Theoretic Framework
}

\author{
Alan W Black \\ Dept of Artificial Intelligence, University of Edinburgh, \\ 80 South Bridge, Edinburgh EH1 1HN, UK. \\ avbeed, ac, uk
}

\begin{abstract}
This paper proposes the use of situation theory as a basic semantic formalism for defining general semantic theories. ASTL, it computational situation theoretic language, is described which goes some way to offering such a system. After a general description of Discourse Representation Theory an encoding of DRT in ASTL is giver. Advantages and disadvantages of this method are then discussed.

Topic: computational formalisms in semantics and discourse
\end{abstract}

\section{Introduction}

The purpose of this paper is to show how a computational language based on situation theory can be used as a basic formalism in which general semantic theories can be implemented. There are many different semantic theories which, because of their different notations, are difficult to compare. A general language which allows those theories to be implemented within it would offer an environment where similar semantic theories could be more easily evaluated.

Situation Theory (ST) has been developed over the last ten years [2]. Much work las been done in both the formal aspects of situation theory and its use in natural language semantics (situation semantics), however so far little work has been done in its computational aspects. It is the eventual goal of the work presented here to show how situation theory can be used computationally and how a computational situation theoretic language can provide an environment in which different semantic theories can be easily compared.

Because there are so many variants of ST we must define our own here, The language ASTL [3] has been defined. Although it uses surprisingly few features of situation theory, it seems powerful enough to act as a basic language for semantics. It has been considered that some extension to "classical" feature structures be made and use those to encode semantic forms. Features systems augmented with set values, cyclicity and other extensions may be powerful enough but the method described here takes an existing semantic theory and refines it rather than building a new one.

This paper is basically split into two sections. The first discusses how ST can be used in a computational system, and introduces the language ASTL The second half of this paper discusses Discourse Representation Theory (IRT) as a theory in itself and shows how it can be encoded with ASTL.

\section{ST and Computation}

The view according to situation theory is that parts of the "world" can be described as situations. Situations support facts. Facts can be true, false, or undefined in some situation. A fact's truth value may be different in different situations. Situations are first class objects in the theory, and hence they can be used as arguments to facts so that relations can be defined between situations. Situations are useful in translations for naked infinitives (e.g. "see"). Situations make S'T different from more conventional logical theories although there have been proposals to add situation-like objects to more classical theories like Montague grammar [8].

As well as situations and partiality, situation theory offers many other intensional objects, including abstractions, types, and parameters (partially determined objects). These form a rich formalism for describing semantic phenomena. However these features alone do not constitute a computational system, with the addition of constraints and rules of inference we call have the basis for a computational system. The idea of a computational situation theoretic language has been considered elsewhere. Most notable is the language Prosit [9] which offers a Prolog-like language based on situation theory rather than first order logic. Other systems (e.g. [5]) allow the representation of situations etc. within some other formalism (e.g. feature structures) but do not use situation theory itself as the basis for the language.

\section{ASTL}

Ast, is a language based on situation theory. It takes a very conservative view of situation theory, admitting only some basic parts. Although AsTL may need to be extended later, it already can be used to describe simple versions of semantic theories (such as situation semantics and DRT). Rather than use, or extend, Prosit it was decided to develop a new language. AsT includes some builtin support for natural language parsing based on the ideas of Situation Theoretic Grammar [4] while Prosit is designed more for knowledge representation than direct language processing.

AsTL allows the following basic terms:

Individuals : e.g. $a, b, c$.

Parameters : e.g. $X, Y, Z$.

Variables: e.g. $* X, * Y, * Z$.

Relations : e.g. see/2. Relation name and arity.

i-terms : consisting of a relation, arguments and a polarity (0 or 1 ), e.g. $\langle<8$ ing, $h, 1\rangle>$. 
types : consisting of an ithstraction over proposi tions. For exanple

$$
\begin{aligned}
& \text { IS } \mathrm{S}: \mathrm{x}\langle\langle\mathrm{ging}, \mathrm{h}, 1\rangle\rangle \\
& \text { S I }=\langle\langle\text { see, h, S, 1)〉] }
\end{aligned}
$$

'['hat is the type of situation which supports the fact that $h$ sings and $h$ sees that situation. Situations : written as names optionally followed by a type. e.g.

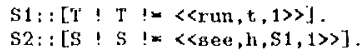

In aldition to terms there are the following sentences:

Propositions : consisting of a situation and it typee.s.

$$
\begin{aligned}
\text { Sit } 1:[S: S: & =\langle<\text { see }, h, S, 1\rangle\rangle \\
S: & =\langle<\text { dance, } h, 1\rangle\rangle]
\end{aligned}
$$

Gonstraints : are defined between propositions, they consist of a proposition following by $<=$ followed by a list of propositions. For example

$$
\begin{aligned}
& \text { Sitt 1: [S ! S != 《happy,h, 1»] }
\end{aligned}
$$

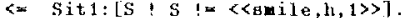

The semantics of $A \mathrm{~S}^{\prime} \mathrm{Y}$, (defined fully in (3]) are de finted in terms of a model consisting of individuals, relations, parameters, situations and a set consistiug of pairs of situations and facts. Informally, it proposition is true if the denotation of the situation supports all of the facts in the type. A constraint is true if when all the propositions in the right hand side of the constraint are true, the left hand propo sition is true also. As it is currently defined AStr. has no built-in definition with respect to coherence, that is there is no built-in mechanism that stops a situalion supporting both a fact and its dual (the fact with the opposite polarity)

Constraints can be generalised using variables. An example will help to illustrate this. If we define the following basic situation and constraint:

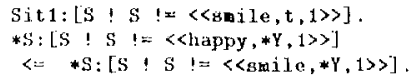

lufornally the constraint states that in any sitiation where something smiles it is also lappy (in that same situation). From the above basic axioms we can derive that the following is true:

$$
\text { Sit } 1:[S \text { I S ! }=\langle\langle h a p p y, t, 1>>
$$$$
\mathrm{S} !=\langle\langle\mathrm{smile}, \mathrm{t}, \mathrm{t}\rangle\rangle]
$$

Rather than just use the linear forms for displaying AST, oljects, an extension las been added for output. Hased on EKN [1] ASTI objects call be displayed as boxes, making complex objects much easier to view. In this notation we write situations as boxes with their tannes in a top left inset with facts written (in a more conventional predicate argument form) inside the box.

Using the work of Cooper [4] we can process language in a situation theoretic way. Situation Theoretic Grammar takes the view that ntterances can be represented by situations. For example

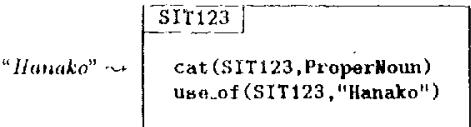

That is, the use of the phrase "Manako" gives rise to a situation that supports the facts that it (the situation) is a Properboum and it is a use of the word "Ianako". We call these utterance situations. As in utterance happens at in particular time and location this fact should also be recorded in the situation. In ASTL this temporal aspect is builtin to the language. A special form of constraint, grammar rules, can be used to constrain utterance situations. General constraints apply to any form of situation (utterance or otherwise) while gram mar rules only apply to utterance situations. $A$ grammar rule between utiterance situations such as

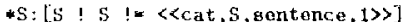

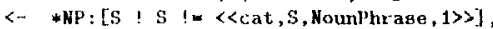

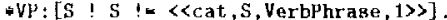

takes into account that the two utterance situations occur next to each other. It is possible to model all of this within the standard constraint system by adding facts about start and end points of utterances (in a similar way that DCG are interpreted in Prolog) but as one of the main uses of AST' is languige processing it was felt more efficient to build utherance situations (and constraints on them) directly into the language.

$\Lambda$ basic implementation has been made within Common Lisp which takes AsтL descriptions (defnitions, hasic situations and constraints) and allows queries to be nude about their sets of constraints and basic situations.

\section{Discourse representation theory}

(Given a simple: laughage like AST there is now the question aljout how it can be used in representing other semautic theories. DRT [7] offers a representation for discourses. A discourse representation stricture (D)RS) is defined at each stage in at discourse describing the current state of the analysis. A DRS consists of two parts; a set of domain markrs, which can be bound to objects introduced into the current discourse, and a set of conditions on these markers. DltSs are typically written as boxes with the markers in the top part and conditions be. low. l'or example a DRS for the utterance " $a$ man sings" is

$\left[\begin{array}{l}x \\ \operatorname{man}(x) \\ \operatorname{sing}(x) \\ -\ldots\end{array}\right]$

'Tle following description of IDRT' in A S'Th is based on the IDRT definition in [6]. First we need a syntactic backbone to be able to discuss the construction of a DRS for a discourse. As seen (briefly) above ASTh offers a basic grammar formalism. That is, granmar rules are specified as constraints betwees utterance situations 
Given such a backbone we need to define an ASTI, representation for DRSs. DRSs have two parts. Discourse markers can be represented as paraneters in ASTL. In situation theory parameters denote partially determined objects. Parameters can be anchored to other objects as information about their denotation is found. DRS conditions are represented by i-terms. A DhS itself is represented as a parametric situation-a situation whose type contains parameters. Discourse markers are not explicitly listed in the DIRS representation. An ASTI, representation of the DRS for "a man sings" is

$$
\begin{aligned}
\text { Sit } 345::[S \quad \text { ! } & !=\langle<\operatorname{man}, X, 1\rangle> \\
& S !=\langle\langle\operatorname{sing}, X, 1\rangle]
\end{aligned}
$$

where $\boldsymbol{X}$ is a parameter.

7 his allows a simple semantics close to that of a conventional DRS. That is an AST DRS will be true for some situation (i.e. a model) if there exists all anchoring for the parameters in it which make it a type of the model-situation. A special definition will be needed for the condition every (and possibly others if extensions to basic ble? are included). It may be better to think of the situation name also as a paraneter which gets anchored to the morlel-situation. But as the semantics of ASTI relates situations names to situations (i.e. two sitwation names can denote the same situation) there is still a level of indirection.

DRSs are objects which are related to utterance situations. They are not themselves representations of the utterances but representations of what the utterances describe.

\section{Threading}

An important aspect of DRT is how a DRS is constructed from a discourse. Here (and in (6]) we use the technique of threading. The general idea is that a DRS gets passed through a discourse being added to as the discourse progresses.

In this description, a discourse consists of a set of utterance situations which can be viewed through a number of different structural relations. The first is through the relation daughter which defines the syntactic structure of the discourse as defined by the grammar rules (immediate dominance ald linear precedence). Secondly the thread relation defines an ordering of the utierances situat ions used in the generation of the DRSS. Lastly there are two relations, range and body used in defining the logical structure of the discoutse.

The threading relation is a binary relation be tween utterance situations. We will say the first. argument is threaded to the second. Fach utterauce situation appears cxactly once as the second argument in the thread relation (i.e. it has exactly one inconing thread). There is one exception, a special situation called DStart which does not have an incoming thread (it is used to represent the mull context at the start of a discourse), but. does appear as an incoming thread for one or more utterance. situations. There are no cycles in threading but as we slall see there nay be more than one linked thread of utterances within a discourse. The actual construction of the threading relations is discussed later.

Lach utterance situation is related to two DJRSs, through the relations DRSIn and DRSDut. A DRSIn DRS is the DRSOut DRS of the incoming thread. 'This constraint can be written in ASTL ats'

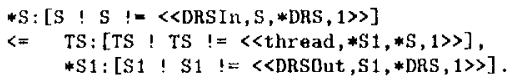

The relation between the two DRSs related to an utterance is also constrained. 'T'his is a core part of DR'. Basically the outgoing DRS contains the same information as the incoming DRS plus any information the utterance adds to the discourse. In the case of a proper noun utterance situation we can capture this relation with the following constraint:

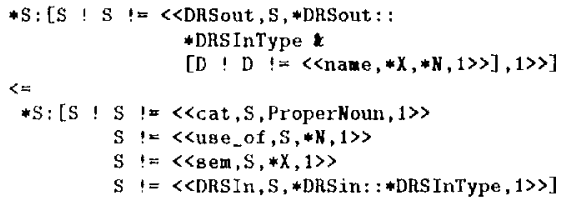

Information is montotonically increasing in DRSs as we traverse along a thread. We are not destructively modifying a DRS as the discourse progresses but constructing a new IORS which supports the same conditions as the incoming DRS. The constraint above forns the outgoing IDRS from the type (*DRSInType) of the incoming one, which will contain all the conditions of the incoming DRS, plus a new condition introducing the parameter for the proper noun and a condition on its name

We also have the constraint that any argument or relation that appears in the conditions of a DKS must be related to some utterance situation by the relation sem previously in that thread. 'This condition means that arguments are threaded before predicates. For example both the subject NI' and object NP of a simple sentence will be threaded before the VP. In contrast in [6] the VP comes before a object NP which means a IDRS is created with an argument in a condition which is not yet determined (i.e a free variable).

The other structural relations are range and body. Each determiner utterance situation appears in exactly one range-relation and exactly one bodyrelation. The second argument to these relations are utterance situations that do not appear as first arguments in any threading relation (i.e. they are ends of threads). 'The DRSOut of a determiner utlerance situation is a function of the DRSIn IDRS plus information from the range and body related threads. In the every determiner case the DRSOut constraint is

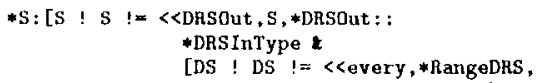




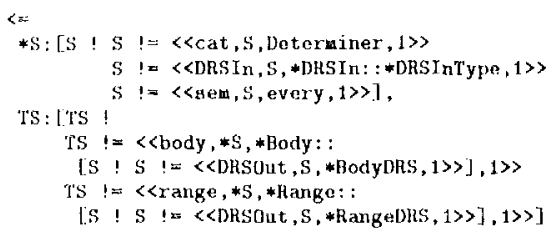

While for the indefinite determiner the DRsout sinply contains all the conditions from the DRSin, range and body related utterances.

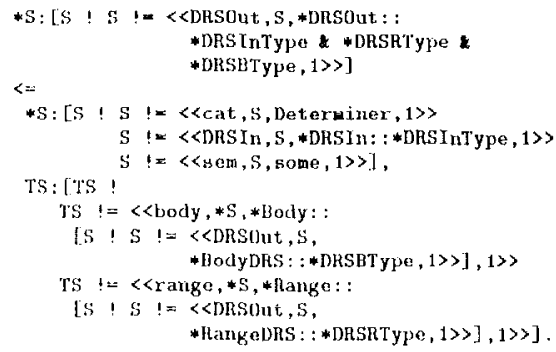

But how is threading huilt? 'l'he grammar rules specify the basic: svotatctic structure (via the daughter relations). At the sume time the threading information can be constructed. Winch utterance situation is related to two others by the relations need and ont. The need relation identilies the utLerance situation (either itself or one of its daughters) which requires an inconing thead while out identifies which situation is to be threaded on to the next part of the discourse. Although the need and out relations are determined at the time a granwar rule is realised the actual thread, range and body relatjous may not be determined locally. The utterance to be threaded to the need of an NP cannot be realised until the NP' is put in context. In contrast with [6] instead of passing up the utterance that neteds a liread, they pass down the "ulterance" that is to be therated in. Ilere we grive a bot.tom ay defintion rather than as in [6] a top down one

As seen in the constrants above the structural facts whose relations are thread, range and body are collected in a situation called TS Helow is an exanple sentence shown as a syntax tree will the threal relation drawn as arrows to show the flow of information through the discourse

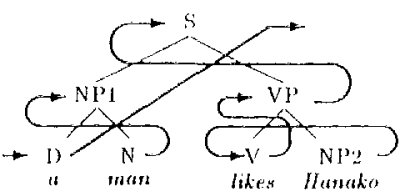

In addition, DStart is thearled to D, H and HP2. The main discourse thread will go through $\mathrm{D}$. There: are two other threads ending at NP1 and S. D will be related to $N P 1$ by the relition range and to $S$ by the relation body. Ilence the mitput DRS from the sentence (from the determiner " $a$ " - by the construints given above) is built from the incoming IDRS plas the ontgoing IDRSs from $\mathrm{Nl}^{2} \mathrm{l}$ and $\mathrm{S}$ (which are related to I) via the range and body relations).

\section{l'ronouns and Accessibility}

Unlike other utterance situations, pronouns do not just adil new information to a DIRS. They also reinure existence of some eferent already introduced in the context. To put it simply there must be a suitable object in the inconing IDIS that the pronoun can match. A condition can be written as

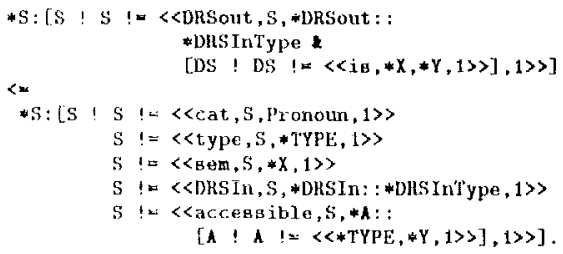

Where *TYPE will be one of rale, female or neuter. Ilowever, it is not sulficient to simply check the conditions in the inconing DRS for some? markin of the right type.

The accessibje relation is also defined over the threading relations. liach utterance situation is refated to a situation that. supports the facts about which matkers are accessible at that point in the discourse. The accessible markers for an utterance situation $V$ are delined (informally) as follows:

If $I !$ is a nom (or propernoun) the accessible markers are from that nom plus the accessible markers fiom the inconing thread.

if $l !$ is the start of a thread whose end is related to a deterniner by the relation body then the aceessible markers are those from the end of that deternimer's range thread.

if $l$ : is the start of a range thread, the accessible: markers are those from the incoming thread of the related determiner.

if $U$ is an indefinite deterniner the arcessible markers are thoss: of the end of the body Mlirecial

if $l$ is an every detenuiner the accessible narkerti are those from its incoming thread (i.e. does not include the markers introduced in l. he range and body threads).

ot.herwise the accessible markers are those of the incoming thread

These conditions can easily be represented by ASTL constirints

Given the above descriptions: a syntactic backbone, a DLLS representation, threadiug and definition for accessibility, we can form IDRSs for simple disconrses. The coverage is that of [6]. 'This still allows an txample of donkey anaphora as in "every man with a donkey likes it". The DhSOut for the discourse utterance situation is. 


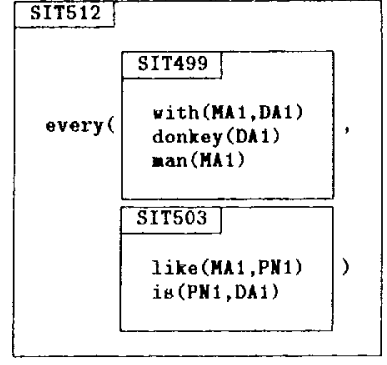

\section{Discussion}

Although translation of DRT into ASTI is possible there are some important consequences. The semantics of an ASTL. DRS, briefly described above, requires that it is possible to tell the properties of every object in the situation. As situations are partial it may not be defined for everything whether it is a man or not, thus it is not possible to define "all men." (Note, lack of information does not imply falsity.) This is perhajes unfair to consider this ats a problem as in the standard definitions of DRT it is recuired that the model be complete (all properties are defined on all objects) - - so it seems no worse to require this of the situation in which we are finding the truth conditions of a DRS. However we could include further definitions for the every relation and require that there be some resource situation that identifies actual objects that fall in its scope. This technique has been used by [1].

There is the question of compositionality. It could be said that the threading relations are only partially determined compositionally. But this seems exactly what the theory states and the intuition behind it. We cannot define a DRS for a noun phrase unless we know what context the NP is in. All that can be determined is partial definition with conditions on the context.

An important aspect of DRT is that there is a left to right dependency on DRSs. This does not necessarily mean that parsing must be left to right, though normally it will be. A definition of IDRT should include this dependency and not rely on how a implementation happens to order processing. The AsTL definition does include a left to right dependency, without specifying a processing order on the inference mechanism.

\section{Summary}

This paper has introduced the notion of using situation theory as a basic formalism in which other semantic theories might be defined. $\Lambda$ computational situation theoretic language called ASTL is discussed. Situation theory is suitable as basis for a metatheory because a representation of situations allows the representation of higher order objects necessary for describing other semantic theories. A possible translation of DRT in $\Lambda \mathrm{ST}$, is given. The coverage is that of [6].

This translation is interesting because first it shows that situation theory is not some opposing semantic theory but that it can be used in discussing other theories. However perhaps it is not surprising that a language such as ASTL is powerful enough to give this translation. A feature system, with sets (or some definition), cycles and constraints is close to what ASTL is, but it is interesting that these properties cau be found as the basis in a current semantic theory without introducing a new theory. Finally a situation theoretic description of DRI allows extensions of DRT to use the properties of situation theory. Situations which are useful in describing various natural language semantic phenomena (e.g. naked infinitives) are now readily available to be included in extensions of DRT.

Acknowledgements: 'This work was supported by an SElRC studentship' award number 89313458 . I would also like to thank Robin Cooper, Ian Lewin and Graeme Ritchic for comments and guidance on this work.

\section{References}

[1] J. Barwise and R. Cooper. Simple Situation Theory and its graphical representation. In Partial and Dynamic Semantics III, DYANA R2.1.C, Cognitive Science, University of Edinburgh, 1991.

[2] J. Barwige and J. Perry. Situations and Attitudes. MIT Press, 1983.

[3] A. Black. A situation theoretic approach to computation semantics. forthcoming $P h D$ thesis, Dept of AI, University of Edinburgh, 1992.

[4] R. Cooper. Information and grammar. Technical Report RP No. 438, Dept of AI, University of Edinburgh, 1989.

[5] J. Fenstad, P-K. Halvorsen, 'T. Langholm, and J. van Bentham. Situations, Lanquage, and Logic. Reidel, Dordrecht, 1987.

[6] M. Johnson and E. Klein. Discourse, anaphora and parsing. In COIING86, Bonn, 1986.

[7] H. Kamp. A theory of truth and semantic representation. In J. Groenendijk, 'I. Janssen, and M. Stokhof, editors, Formal Methods in the Siudy of Language. Mathematical Center, Amsterdam, 1981.

[8] R. Muskens. Meaning and Partiality. PhD thesis, University of Amsterdam, 1989.

[9] H. Nakashima, H. Suzuki, P-K. Halvorsen, and $S$. Peters. Towards a computational interpretation of situation theory. In FGCS, ICOT, 1988. 